\title{
INTERFACIAL MOTION IN SHAPE MEMORY ALLOYS
}

\author{
M. BUISSON, E. PATOOR and M. BERVEILLER \\ Laboratoire de Physique et Mécanique des Matériaux, U.R.A. C.N.R.S. $N^{\circ} 1215$, Institut Supérieur \\ de Génie Mécanique et Productique, Ile du Saulcy, F-57045 Metz cedex 1, France
}

\begin{abstract}
We investigate interfacial motions between martensitic variants. Analytically, we describe simply the topology and kinematics of a representative volume constituted of several compatible variants whose interfaces have a velocity differing from those of the material particles. The corresponding driving force is deduced using Eshelby's energy momentum formalism and compared with a critical threshold from which we define a reorientation criterion. The interface movement is taken into account via the concept of an inclusion with moving boundary and we propose a formulation of the global behaviour.
\end{abstract}

\section{1.- Introduction,}

Interfacial motions are at the origin of many physical mechanisms which are responsible for inelastic behaviour. Twinning, phase transformations or some aspects of the classical plasticity like shear banding are related to this phenomena.

In this work, we investigate the particular case of materials undergoing displacive phase transformations. Such transitions happen without diffusion phenomena and require weak rearranging at the atomic scale. Symmetries of the crystal lattice allow the view of several martensitic variants; such variants are domains where stress free transformation strains are almost uniform, they differ from one another in crystallographic orientation. Their number and nature depend on the thermomechanical loading and microstructures of the material. We look at the case for which the inner mechanism of the macroscopic strain is due to interfacial movements with a velocity differing from that of the material particles.

Globally, this phenomenon is called reorientation for which the volume of a variant (I) increases to the detriment of a neighbouring variant (J); in such a case we observe the movement of the interface (IJ) between variant $(I)$ and $(J)$.

We propose a general form for the macroscopic behaviour law through a kinematical description of strains and through the determination of the thermodynamic forces which are responsible for the motion of interfaces. The calculation of such forces is performed using Eshelby's energy momentum formalism, we assume small strains, compatible interfaces (e.g. no internal stresses are induced). We define a yield surface and the use of plastic-strain potential allowes us to formulate the constitutive relation for such a category of materials and mechanisms. This analysis is based on simple topological and kinematical arguments from which we bring out microstructural parameters of the global behaviour.

\section{2.- Some topology and kinematics.}

At first, we consider the topology of a representative volume $\mathrm{V}$ constituted of $\mathrm{N}$ martensitic variants. We assimilate each variant with a boundary moving inclusion (geometric volume $\mathrm{V}_{\mathrm{I}}$, volume fraction $\mathrm{f}$, 
boundary $\partial V_{I}, I=1$ to $N$ ) embedded in the volume $V$ and bordered by others variants $V_{J}$ so that we can recompose $\partial \mathrm{V}_{\mathrm{I}}$ as the union (symbol $\mathrm{U}$ ) of elementary surfaces $\partial \mathrm{V}^{\mathrm{IJ}}$ :

(1) $\quad \partial V_{1}=\bigcup_{J=1}^{N} \partial V^{I J}$

where $\partial \mathrm{V}^{\mathrm{IJ}}$ constitutes the interface (IJ) between variants $(\mathrm{I})$ and $(\mathrm{J})$, we consider in this union that $\partial \mathrm{V}^{\mathrm{IJ}}$ is zero if $V_{I}$ and $V_{J}$ are not adjoining.

Kinematically, it is crucial to point out that each interface (IJ) moves with a velocity which is different from those of the material particles: the mobility of interfaces is taken into account via the concept of the inclusion-problems with moving boundaries $/ 1 /, 2 /$. We use the technic of time derivation $\delta / \delta$ t with respect to the vector field $\underline{W}(\underline{x}, t)$ defined by the eigen-velocities of the interfaces (e.g. velocities at time $t$ of geometrical points of volume $V_{I}$ with cartesian coordinates $x$ in a reference system from which we observe the movements of interfaces $\partial \mathrm{V}^{\mathrm{IJ}}$ ):

$$
\text { (2a) } \frac{\delta}{\delta t}()=\frac{\partial}{\partial t}()+\underline{w} \cdot \operatorname{grad}() \quad(2 b) \frac{\delta}{\delta t} \iiint_{V(c)}() d \underline{x}=\iiint_{V(t)} \frac{\partial}{\partial t}() d \underline{x}+\iint_{\partial V}() \underline{w} \cdot \underline{n} d \underline{a}
$$

Assuming that $\underline{\mathrm{W}}$ is approximately uniform for each section $\partial \mathrm{V}^{\mathrm{IJ}}$, we define the velocity $\underline{\mathrm{W}}^{\mathrm{IJ}}$ and surface vector $\underline{S}^{I J}$ respectively:

(3) $\quad \underline{\mathrm{w}}(\underline{\mathrm{a}}, \mathrm{t})=\underline{\mathrm{w}}^{\mathrm{IJ}}(\mathrm{t})$ for $\underline{\mathrm{a}} \in \partial \mathrm{V}^{\mathrm{IJ}}, \mathrm{s}_{\mathrm{i}}^{\mathrm{I}}\langle\mathrm{t})=\iint_{\partial V^{J}} \mathrm{n}_{\mathrm{i}}(\mathbf{a}, \mathrm{t}) \mathrm{d} \underline{\mathrm{a}}$

with $S^{I J}{ }_{i}=-S_{i} I_{i}$ due to the convention of the unit external normal $\underline{n}$ and $S^{\mathrm{IJ}} \mathrm{i}_{\mathrm{i}}=0$ if $\mathrm{V}_{\mathrm{I}}$ and $\mathrm{V}_{\mathrm{J}}$ are not adjoining or $(\mathrm{I})=(\mathrm{J})$ or $\partial \mathrm{V}^{\mathrm{IJ}}$ on the external boundary $\partial \mathrm{V}$.

Let us note that $\left(\mathrm{S}_{i}^{\mathrm{IJ}} \mathrm{S}_{i}{ }^{\mathrm{IJ}}\right)^{1 / 2} / \mathrm{V}$ (with summation convention on the repeated indices) is the surface of interface $\partial V^{I J}$ per unit volume, this parameter plays a leading part in the evolution of the microstructure.

In the specific case of the reorientation of martensitic variants, we assume that each variant (I) has at time $t$ a volume $V_{I}(t)$ with a varying size and morphology; nevertheless, in each variant $(I)$, the characteristic physical and mechanical properties (.... I are assumed uniform and time-constant. (2b) becomes

$$
\frac{\delta}{\delta \mathrm{t}} \iiint_{\mathrm{V}}(\quad) \mathrm{d} \underline{\mathrm{x}}=\sum_{\mathrm{I}=1}^{\mathrm{N}} \frac{\delta}{\delta \mathrm{t}} \iiint_{\mathrm{V}_{\mathrm{I}}} \int_{\mathrm{I}}() \mathrm{d} \underline{\mathrm{x}}=\sum_{\mathrm{I}=1}^{\mathrm{N}}(\quad)^{\mathrm{I}}\left[\sum_{\mathrm{J}=1}^{\mathrm{N}} \mathrm{w}_{\mathrm{k}}^{\mathrm{II}} \mathrm{s}_{\mathrm{k}}^{\mathrm{VI}}\right]
$$

Using the symmetry properties of $S^{I J_{i}}$, it is possible to consider in (4) all discontinuities $(\ldots . .)^{I}-(\ldots . .)^{J}$ (e.g. jump of a field quantity (.....) across $\partial \mathrm{V}^{\mathrm{II}}$ ):

$$
\text { (5) } \quad \frac{\delta}{\delta \mathrm{t}}(\cdot)=\sum_{\mathrm{J}=1}^{\mathrm{N}-1} \sum_{\mathrm{J}>\mathrm{A}}^{\mathrm{N}}\left[()^{\mathrm{I}} \cdot(\quad)^{\mathrm{J}}\right] \mathrm{w}_{\mathrm{k}}^{\mathrm{J}} \mathrm{S}_{\mathrm{k}}^{\mathrm{S}}
$$

For example, from (4) we deduce the rate of volume-fraction $\mathrm{f}^{\mathrm{I}}=\mathrm{V}_{\mathrm{I}} / \mathrm{V}$ of variant $(\mathrm{I})$

$$
\text { (6) } \frac{\delta}{\delta \mathrm{t}} \mathrm{f}^{\mathrm{I}}=\sum_{\mathrm{J}=\mathrm{I}}^{\mathrm{N}} \mathrm{w}_{\mathrm{i}}^{\mathrm{IJ}} \mathrm{s}_{\mathrm{i}}^{\mathrm{IJ}} / \mathrm{V}
$$

where the global variation $(\delta \mathrm{V} / \delta \mathrm{t}) / \mathrm{V}^{2}$ is assumed negligible. In the same way we deduce from (5) the 
rate of total global strain $E(t)$ which is the volume average of the linearized total local strain $\varepsilon(x, t)$ :

$$
\frac{\delta}{\delta t} \mathrm{E}=\sum_{\mathrm{l}=1}^{\mathrm{N}-1} \sum_{\mathrm{t}>\mathrm{x}}^{\mathrm{N}}\left(\varepsilon^{\mathrm{I}}-\varepsilon^{\mathrm{J}}\right) \mathrm{W}_{\mathrm{k}}^{\mathrm{I}} S_{\mathrm{k}}^{\mathrm{I}} / \mathrm{V}
$$

which is written in terms of jumps $\left(\varepsilon^{\mathrm{I}}-\varepsilon^{\mathrm{J}}\right)$ and interface movements $\mathrm{W}^{\mathrm{IJ}}{ }_{\text {. }}$. In the following, we look at the thermodynamic force associated to this movement.

\section{3.- Interfacial driving force - Global behaviour.}

a) Energy momentum tensor-Interfacial force:

Eshelby $/ 3 /$ introduced the energy momentum tensor in solids mechanics; he investigated the evolution $\delta \Phi / \delta$ t of the total potential energy in case of the movement of an interface. Hill /4/ extended this notion by using the principle of virtual works associated with an arbitrary defined displacement field $\underline{w}(\mathrm{x}, t)$.

In our work, we examine the rate of total potential energy $\delta \Phi^{\mathrm{IJ}} / \delta \mathrm{t}$ when a variant $\mathrm{V}_{\mathrm{I}}$ (inclusion) develops to the detriment of an other variant $\mathrm{V}_{\mathrm{J}}$ (matrix) with respect to the following restrictions:

- we assume the compatibility of variants and homogeneous elasticity: the Cauchy-stress is uniform $\left(\left(\sigma_{i j}(\underline{x}, t)=\Sigma_{i j}(t)\right)\right.$ where $\Sigma_{i j}$ are the components of the macroscopic applied stress $)$ and discontinuities of strain-energy density between variants become zero.Consequently, the jump $\varepsilon-\varepsilon$ of total strains may be replaced by $\varepsilon^{\mathrm{tI}}-\varepsilon^{\mathrm{tJ}}$ where $\varepsilon^{\mathrm{t}}$ is the stress-free transformation strain.

- finally the hypothesis of uniform properties inside each variant lead us to cancel here gradients without distinction from lagrangian or eulerian coordinates. Then following Hill /4/, we use directly formula (51) and (60) of /4/ to obtain:

$$
\frac{\delta}{\delta t} \Phi=\Sigma_{i j}\left(\varepsilon_{i j}^{\mathrm{U}}-\varepsilon_{\mathrm{ij}}^{\mathrm{t}}\right) w_{k}^{\mathrm{II}} s_{k}^{\mathrm{y}}
$$

so that the associated driving force is $(/ 3 /)$ :

$$
F^{\mathrm{IJ}}=\Sigma_{\mathrm{ij}}\left(\varepsilon_{\mathrm{ij}}^{\mathrm{II}}-\varepsilon_{\mathrm{ij}}^{\mathrm{iJ}}\right)
$$

Carrying on this work, the description of the global behaviour law depends unavoidably on the intrinsic behaviour of the interfaces. We consider here that the interface will move if the force FIJ (analogous to a resolved stress) comes to a certain critical threshold force $F_{c}{ }_{c}$ which depends in a quite complicated way from the internal structural parameters and volume fractions $\mathrm{f}^{\mathrm{K}}$.

b) Interface behaviour:

We define a yield surface $\mathrm{G}^{\mathrm{IJ}}\left(\Sigma_{\mathrm{ij}}, \mathrm{f}^{\mathrm{K}}\right)=\mathrm{F}^{\mathrm{IJ}}-\mathrm{F}_{\mathrm{c}}^{\mathrm{IJ}}\left(\mathrm{f}^{\mathrm{K}}\right)$. From (9) we observe that

$$
\frac{\partial G^{\amalg}}{\partial \Sigma_{i j}}=\varepsilon_{i j}^{I}-\varepsilon_{i j}^{J}=\varepsilon_{i j}^{I}-\varepsilon_{i j}^{J}
$$

so that (7) becomes

$$
\text { (11) } \quad \frac{\delta}{\delta \mathrm{t}} \mathbf{E}=\sum_{\mathrm{I}=1}^{\mathrm{N} \cdot \mathrm{L}} \sum_{\mathrm{D} D \mathrm{I}}^{\mathrm{N}} \frac{\partial \mathrm{G}^{\mathrm{UI}}}{\partial \Sigma} \mathrm{w}_{\mathrm{k}}^{\mathrm{U}} s_{\mathrm{k}}^{\mathrm{U}} / \mathrm{V}
$$

Using the consistency condition $/ 5 / \delta \mathrm{G}^{\mathrm{IJ}} / \delta \mathrm{t}=0=\delta \mathrm{F}^{\mathrm{IJ}} / \delta \mathrm{t}-\delta \mathrm{F}_{\mathrm{c}} \mathrm{U} / \delta \mathrm{t}$, it remains now to substitute 
$\mathrm{W}^{\mathrm{IJ}} \mathrm{k}^{\mathrm{IJ}}{ }_{\mathrm{k}}$ in term of $\delta \Sigma / \delta \mathrm{t}$. From (9) we deduce $\delta \mathrm{F}^{\mathrm{IJ}} / \delta \mathrm{t}$ and by composition we have successively from (6):

$$
\begin{aligned}
& \frac{\delta F_{c}^{\mathrm{J}}}{\delta t}=\sum_{\mathrm{K}=1}^{N} \frac{\partial \mathrm{F}_{\mathrm{c}}^{\mathrm{J}}}{\partial \mathrm{f}^{\mathrm{K}}} \cdot \frac{\delta \mathrm{f}^{\mathrm{K}}}{\delta \mathrm{t}}=\sum_{\mathrm{K}=1}^{\mathrm{N}} \sum_{\mathrm{L}=1}^{N} \frac{\partial F_{c}^{\mathrm{J}}}{\partial \mathrm{f}^{\mathrm{K}}} w_{i}^{\mathrm{KI}} s_{i}^{\mathrm{KL}} / \mathrm{V} \\
& \text { (12) } \\
& =\sum_{\mathrm{X}=1}^{\mathrm{N}-1} \sum_{\mathrm{L} \rightarrow \mathrm{K}}^{\mathrm{N}}\left(\frac{\partial}{\partial \mathrm{K}} \mathrm{F}_{\mathrm{c}}^{\mathrm{II}}-\frac{\partial}{\partial \mathrm{f}} \mathrm{F}_{\mathrm{c}}^{\mathrm{JJ}}\right) \mathrm{W}_{\mathrm{i}}^{\mathrm{KL}} \mathrm{s}_{\mathrm{i}}^{\mathrm{KL}} / \mathrm{V}
\end{aligned}
$$

with use of the antisymmetry property of $S^{\mathrm{KL}}$.

so that the consistency condition becomes

$$
\left(\varepsilon_{i j}^{\mathrm{II}}-\varepsilon_{\mathrm{ij}}^{\mathrm{U}}\right) \frac{\delta}{\delta \mathrm{t}} \Sigma_{\mathrm{ij}}=\sum_{\mathrm{K}=1}^{\mathrm{N}-1} \sum_{\mathrm{L}>\mathrm{K}}^{\mathrm{N}}\left(\frac{\partial}{\partial \mathrm{f}^{\mathrm{K}}} \mathrm{F}_{\mathrm{c}}^{\mathrm{J}}-\frac{\partial}{\partial \mathrm{f}^{\mathrm{L}}} \mathrm{F}_{\mathrm{c}}^{\mathrm{J}}\right) \mathrm{W}_{1}^{\mathrm{KL}} \mathrm{s}_{1}^{\mathrm{KL}} / \mathrm{V}
$$

Let us define the square-matrix A with upper indices(IJ),(KI)by

$$
A^{(D)(K L)}=\left(\frac{\partial}{\partial \mathrm{f}^{\mathrm{K}}} F_{c}^{J} \cdot \frac{\partial}{\partial \mathrm{f}^{\mathrm{L}}} \mathrm{F}_{\mathrm{c}}^{\mathrm{I}}\right) \quad(\mathrm{J}>\mathrm{I}, \mathrm{L}>\mathrm{K})
$$

which is assumed to have generally an inverse (each pair (IJ) and (KL) stands for an index-number which identifies the interface (IJ) and (KL)). Eliminating $\underline{\mathrm{W}}^{\mathrm{KL}}, \underline{\mathrm{S}}^{\mathrm{KL}}$ and $\varepsilon^{\mathrm{tI}}-\varepsilon^{\mathrm{tJ}}$ we obtain a gene ralized macroscopic flow rule

$$
\frac{\delta}{\delta t} E_{i j}=\sum_{\mathrm{I}=1}^{N-1} \sum_{J>I}^{N} \sum_{\mathrm{K}=1}^{N-1} \sum_{\mathrm{l}>\mathrm{K}}^{N} \frac{\partial G^{I J}}{\partial \Sigma_{\mathrm{ij}}}\left[A^{-1}\right]^{(\text {(J)(KL) }} \frac{\partial G^{\mathrm{KL}}}{\partial \Sigma_{\mathrm{kl}}} \frac{\delta}{\delta \mathrm{t}} \Sigma_{\mathrm{kl}}
$$

In this results, the matrix $A(\mathrm{IJ})(\mathrm{KL})$ is analogous to a hardening matrix from which we take into account the influence of the evolution of the substructures on the critical stresses.

The knowledge of this matrix is lying with a precise description of the material structure (topology of interfaces) and with their critical intrinsic resolved stresses.

\section{References.}

/1/ SABAR, H.,BERVELLER, M.and BUISSON, M., Problème d'inclusion à frontière mobile,C.R.Acad Sci Paris, 1990 , t.310 série II, pp. 447-452.

12/ SABAR, H., BUISSON, M.and BERVEILLER, M., The Inhomogeneous and Plastic Inclusion Problem with Moving Boundary, Inter. Journal of Plasticity, éd Pergamon, in press.

13/ ESHELBY, J.D., Inelastic Behaviour of Solids, éd : M.F. KANNINEN, W.F. ADLER, A.R. ROSENFIELD and R.I. JOFFEE, p 77, Mac Graw Hill, New York 1970.

14/ HILL, R., Energy-Momentum Tensors in Elastostatics : some reflections on the general theory, $\underline{\text {. }}$ Mech. Phys. Solids, 1986, Vol 34, $\mathrm{N}^{\circ} 3$, pp. 305-317.

15/ J.LEMAITRE, J.L. CHABOCHE, Mécanique des matériaux solides, Dunod, Paris, 1988. 